\title{
A Novel Variable-Length Header Extraction Scheme Based on Ring Laser for All-Optical Packet Switching Network
}

Dariush Jafari ( $\nabla$ d_jafari@semnan.ac.ir )

Semnan University https://orcid.org/0000-0003-1033-9991

Mohammad Danaie

Semnan University

Pejman Rezaei

Semnan University

Tofiq Nurmohammadi

Tabriz University: University of Tabriz

\section{Research Article}

Keywords: Header extraction, rate equations, Contrast ratio, All-optical devices

Posted Date: May 11th, 2021

DOI: https://doi.org/10.21203/rs.3.rs-493450/v1

License: (c) (i) This work is licensed under a Creative Commons Attribution 4.0 International License. Read Full License 


\title{
A Novel Variable-Length Header Extraction Scheme Based on Ring Laser for All-Optical Packet Switching Network
}

\author{
Dariush Jafari ${ }^{\mathrm{a}}$, Mohammad Danaie ${ }^{\mathrm{a}}$, Pejman Rezaei ${ }^{\mathrm{a}}$ and Tofiq Nurmohammadi ${ }^{\mathrm{b}}$ \\ ${ }^{\text {a }}$ Electrical and Computer Engineering Faculty, Semnan University, Semnan, Iran \\ ${ }^{\mathrm{b}}$ Department of Electrical and Computer Engineering, University of Tabriz, Tabriz, 5166616471, Iran
}

\begin{abstract}
:
In this paper, a novel header extraction scheme based on semiconductor ring laser is proposed and simulated for proof of principle. This scheme is to be used in all-optical packet switching network which can extract return-to-zero (RZ) format modulation data packet with variable header/payload length and intensive spacing. Simulations and numerical analysis for a 3-byte header and 128-byte payload show that this structure can extract the headers with more than $20 \mathrm{~dB}$ contrast ratio. Additionally, the structure simplicity and its small size is proper candida to integration in alloptical circuits.
\end{abstract}

Keywords: Header extraction, rate equations, Contrast ratio, All-optical devices.

\section{Introduction}

Today's optical technologies developed in many aspects and branches [1-6]. The rapid growth of the internet, increasing data traffic and all-optical processing and storages in the modern societies force the communication networks to increase the bandwidth and capacity [7-9]. Hence, it is preferred to use optical communication networks that work based on optical packet switching (OPS) instead of current optical packet circuits (OPC) networks [10, 11]. One of the main restrictions of the current networks is electronic-optical-electronic conversion (EOE) which is used for packet identification and processing in the electronic domain. Packets in optical communication networks consist of a header and payload [12]. One of the essential functions in the OPS switch is the separation of the packet header from the optical packet stream $[13,14]$. Up to now, several different methods have been proposed in order to extract and process of the packet header. These methods depended on labeling techniques. In the serial labeling techniques, the label is placed to the payload in the serial form and in parallel labeling techniques the label is processed parallel with the payload. There are some challenges in the parallel labeling techniques such as the label multiplexing on a separate wavelength or on a subcarrier frequency [14-18]. In the parallel labeling techniques speed of extracting label is high but packet requires more bandwidth so, in this method the bandwidth is occupied [19]. Intensity modulated payload as well as frequency shift keying (FSK) or Binary phase shift keying (BPSK) label or at different label code are serial labeling techniques [20-24]. These methods are amplitude or phase depended and require synchronization pulse and need fixed packet data length hence, these methods not suitable for flexible optical networks. 
Since ring laser can be used in the design of various optical gates as well as in data storage [25] and processing [24], it is an appropriate candidate to be used in the design of all-optical integrated circuit $[25,26]$. In this paper, a structure based on ring laser is proposed which is capable of separating RZ coded headers from the packet, with high speed, and without delay.

The organization of this paper is as follow. The proposed structure is introduced in section 2 . Section 3 is devoted to calculations and analysis of the proposed structure. In section 4, simulation results will be discussed and finally, section 5 concludes the paper.

\section{Header extraction structure}

Figure 1 shows a simple scheme of the proposed header extractor. In this structure, continues wave (CW) laser beam and packet stream signal are applied into port A and B, respectively. Then the separated header signal and its inverse exit from port $\mathrm{C}$ and $\mathrm{D}$ respectively.

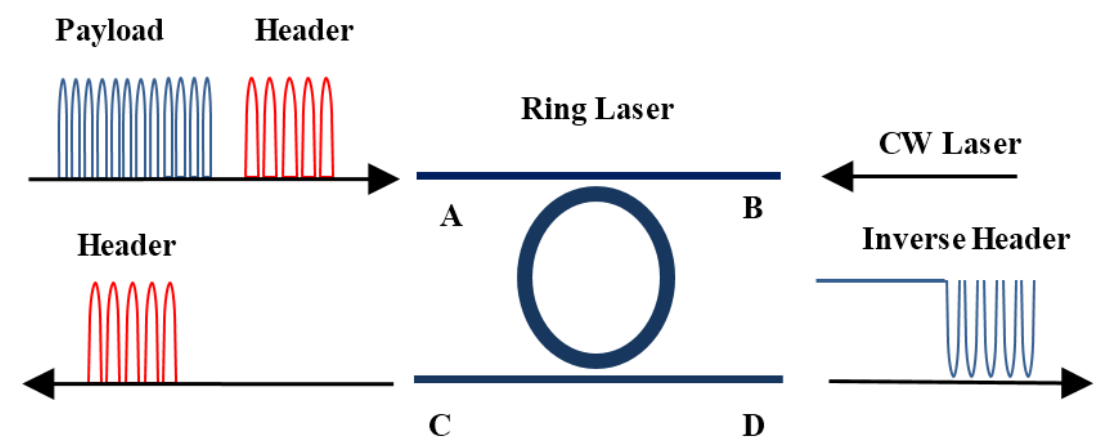

Figure 1. Schematic diagram of optical header extraction

In the first case, assume that the packet signal does not exist, and the laser beam is applied. In this situation, the beam inside the ring propagates clockwise and results the signal at port $\mathrm{D}$ and the zero signal at port $\mathrm{C}$ and.

In the second case, it is supposed that the header with $15 \mathrm{~Gb} / \mathrm{s}$ bitrate is applied into port A. Assuming that the power of input header stream is higher than the laser beam, and the length of header's Gaussian pulse is long, these pulses saturate the ring laser and invert the beam propagation direction inside the ring and cause the packets to appear at port $\mathrm{C}$.

Note that if bitrate of the packets that applied into port B increases, the pulse length decreases, hence it cannot change the propagation direction inside the ring, and the packets will not appear at port C. 


\section{Modeling and numerical analysis}

Ring laser is an active device consisting of one ring and two waveguides. Applying current to the active region causes the lasing operation. in the initial state, when the current amplitude is low, the linear characteristic is dominant, and both clockwise $(\mathrm{CW})$ and counter-clockwise $(\mathrm{CCW})$ modes can propagate inside the ring. Increasing the applied current causes the domination of the nonlinear characteristics which leads to the ring laser operate in the unidirectional mode, and only one mode can propagate inside the ring [27-29].

The longitudinal electric fields inside the ring cavity are expressed as:

$$
\begin{aligned}
& E(x, t)=E_{1}(t) \exp \left[-i\left(\omega_{01} t-k x\right)\right]+E_{2}(t) \exp \left[-i\left(\omega_{01} t-k x\right)\right] \\
& E_{\text {ext }}(x, t)=E_{\text {ext }}(t) \exp \left[-i\left(\left(\omega_{02} t+\Delta \omega_{\text {ext }} t+\Delta \phi_{\text {ext }}\right)+k x\right)\right]
\end{aligned}
$$

Where $\mathrm{E}_{1}(\mathrm{t}), \mathrm{E}_{2}(\mathrm{t})$, and $\mathrm{E}_{(\mathrm{ext})}$ are mean complex electrical field corresponding to $\mathrm{CW}, \mathrm{CCW}$, and external injection amplitude modes, respectively. $\omega_{1}$ and $\omega_{2}$ are optical frequencies of ring cavity modes in clockwise and counter-clockwise respectively, and $\mathrm{x}$ represents the longitudinal spacing. $\Delta \omega_{\text {ext }}$ is detuning angular frequency of external injection and free running mode, and $\Delta \Phi$ is their phase difference. The time evolution of the fields inside the cavity can be described by the following the rate equations $[30,31]$ :

$$
\begin{aligned}
& \frac{d E_{1}}{d t}=\frac{1}{2}(1-i \alpha)\left(\Gamma v_{g} g_{n}\left(N-N_{t r}\right) \times\left(1-\varepsilon_{s}\left|E_{1}\right|^{2}-\varepsilon_{c}\left|E_{2}\right|^{2}\right)-\frac{1}{\tau_{p 1}}\right) E+ \\
& i\left(\omega_{01}-\omega_{t h}\right) E_{1}+K_{e x t} E_{e c w} \exp \left[-i\left(\Delta \omega_{e x t} t+\Delta \phi_{e x t}\right)\right] \\
& \frac{d E_{2}}{d t}=\frac{1}{2}(1-i \alpha)\left(\Gamma v_{g} g_{n}\left(N-N_{t r}\right) \times\left(1-\varepsilon_{s}\left|E_{1}\right|^{2}-\varepsilon_{c}\left|E_{2}\right|^{2}\right)-\frac{1}{\tau_{p 1}}\right) E_{2} \\
& +i\left(\omega_{01}-\omega_{t h}\right) E_{2}+K_{e x t} E_{e c c w} \exp \left[-i\left(\Delta \omega_{e x t} t+\Delta \phi_{e x t}\right)\right]
\end{aligned}
$$

Where spatial overlap between optical mode and active layer gain represent by optical confinement factor $\Gamma . g_{n}$ and $v_{g}$ are differential gain and group velocity in the active layer, respectively. $\alpha$ is the linewidth enhancement factor in the semiconductor medium which is related to phase-amplitude coupling. $\mathrm{N}$ is carrier density, while $\mathrm{N}_{\text {th }}$ and $\omega_{\text {th }}$ are carrier density and longitudinal resonant frequency at transparency, respectively. $\tau_{1}$ and $\tau_{2}$ are photon lifetime in the ring cavity of the two modes. $K_{\text {ext }}=\sqrt{ }\left(T_{r} / f_{b}\right)$ is the coupling coefficient in which $T_{r}$ is the power coupling rate, and $f_{b}$ is feedback power rate. $\varepsilon_{\mathrm{s}}$ and $\varepsilon_{\mathrm{c}}$ are self-saturation coefficient and cross gain saturation coefficient, respectively. In these equations, the carrier density in the active region is defined as [33]: 


$$
\begin{aligned}
& \frac{d N}{d t}=\frac{j}{e V}-\frac{N}{\tau_{e}}-\left(v_{g} g_{n}\left(N-N_{t r}\right) \times\left(1-\varepsilon_{s}\left|E_{1}\right|^{2}-\varepsilon_{c}\left|E_{2}\right|^{2}\right)\right)\left|E_{1}\right|^{2} \\
& -\left(v_{g} g_{n}\left(N-N_{t r}\right) \times\left(1-\varepsilon_{s}\left|E_{2}\right|^{2}-\varepsilon_{c}\left|E_{1}\right|^{2}\right)\right)\left|E_{2}\right|^{2}
\end{aligned}
$$

Where $\mathrm{V}$ is the volume of the quantum well active region, $\mathrm{j}$ is the injection current density, $\mathrm{e}$ is the electron charge, and finally, $\mathrm{t}_{\mathrm{e}}$ is the carrier lifetime.

\section{Results and Discussions}

One of the most important parameters to define the header extractor performance is contrast ratio (CR) as defined by equation 6 in which $\mathrm{N}$ and $\mathrm{M}$ are the number of payload and header pulse, respectively. Also, $\mathrm{P}_{\mathrm{H}}$ and $\mathrm{P}_{\mathrm{P}}$ represent header and payload power, respectively [18].

$$
C R=10 \log \frac{\frac{1}{N} \sum_{j=1}^{N} P H}{\frac{1}{M} \sum_{j=1}^{M} P L}(d B)
$$

For simulating of the proposed structure, the Gaussian pulse is applied to the ring laser. In order to create the packet sequence, 2-bit header random data was combined with 20-bit payload stream. Also, based on experimental parameters in [27-31], the active region of the ring laser is $\mathrm{InGaAs}$ and its radius is assumed $6 \mu \mathrm{m}$. The simulation parameters are listed in Table 1.

Table 1. Ring laser simulation parameters.

\begin{tabular}{|c|l|l|}
\hline Symbol & \multicolumn{1}{|c|}{ Description } & \multicolumn{1}{c|}{ Value } \\
\hline $\mathrm{R}$ & Ring Radius & $6 \mu \mathrm{m}$ \\
\hline$\Gamma$ & Confinement Factor & 0.226 \\
\hline$\alpha$ & Linewidth Enhancement Factor & 4 \\
\hline $\mathrm{g}_{\mathrm{n}}$ & Group Velocity & $0.857 \mathrm{e}-8 \mathrm{~m}^{\prime} \mathrm{s}^{-1}$ \\
\hline$\tau_{\mathrm{p} 1}$ & Photon Lifetime for Mode1 & $4.85 \mathrm{e}^{-12} \mathrm{~s}$ \\
\hline$\tau_{\mathrm{p} 2}$ & Photon Lifetime for Mode2 & $4.85 \mathrm{e}^{-12} \mathrm{~s}$ \\
\hline$\tau_{\mathrm{p}}$ & Photon Lifetime & $4.85 \mathrm{e}^{-12} \mathrm{~s}$ \\
\hline$\tau_{\mathrm{e}}$ & Electron Lifetime & $3.14 \mathrm{e}^{-9} \mathrm{~m}^{2} \mathrm{~s}$ \\
\hline $\mathrm{T}$ & Coupler Transmission & 0.3 \\
\hline$f_{\mathrm{b}}$ & Power Coupling Back Ratio & 0.5 \\
\hline$\varepsilon_{\mathrm{s}}$ & Self-nonlinear Coefficient & $3.16 \mathrm{e}^{-23} \mathrm{~m}^{3}$ \\
\hline$\varepsilon_{\mathrm{c}}$ & Cross-nonlinear Coefficient & $6.32 \mathrm{e}^{-23} \mathrm{~m}^{3}$ \\
\hline $\mathrm{I}$ & Bias Current & $3 \mathrm{~mA}$ \\
\hline$\lambda$ & Lasing Wavelength & $1.55 \mu \mathrm{m}$ \\
\hline $\mathrm{N}_{\mathrm{th}}$ & Carrier Density at Transparency & $0.788 \mathrm{e}^{23} \mathrm{~m}^{-3}$ \\
\hline $\mathrm{P}_{\mathrm{cw}}$ & Input laser(CW)power & $1 \mathrm{~mW}$ \\
\hline
\end{tabular}


Figure 2 shows the output propose scheme with the packet stream contained 2-byte header and 128-byte payload random data stream input. As can be seen, the header stream has been amplified while the payload bits have been attenuated. Additionally, CR of the output header is greater than $18 \mathrm{~dB}$. Also, figure 3 represented the output of header extractor with 3-byte and 128-byte random data stream input. It is clear that the size of data packet and space between header and payload don't effect to the output result of the proposed scheme.

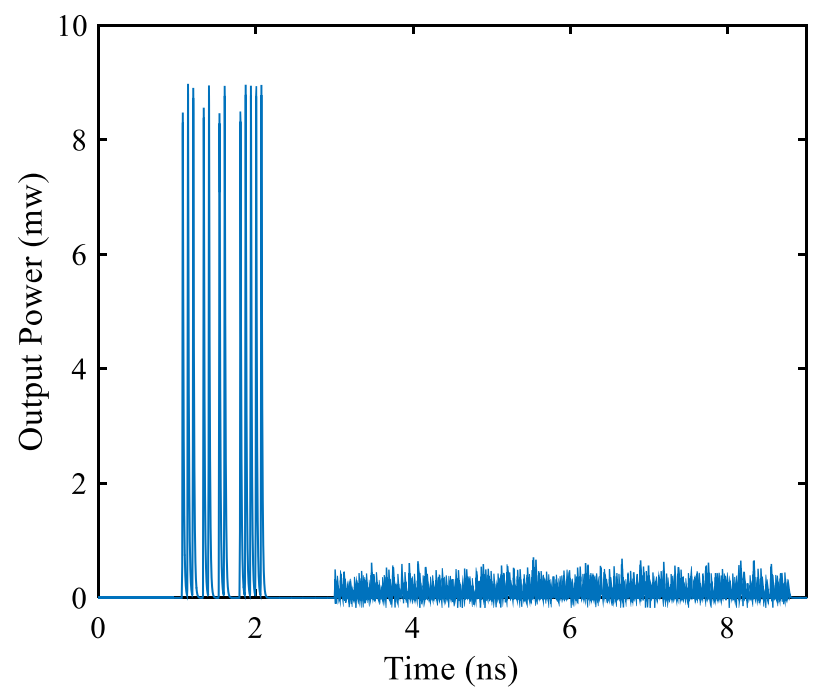

Figure 2. Output power packet extractor with 2-byte header and 128-byte payload input packet.

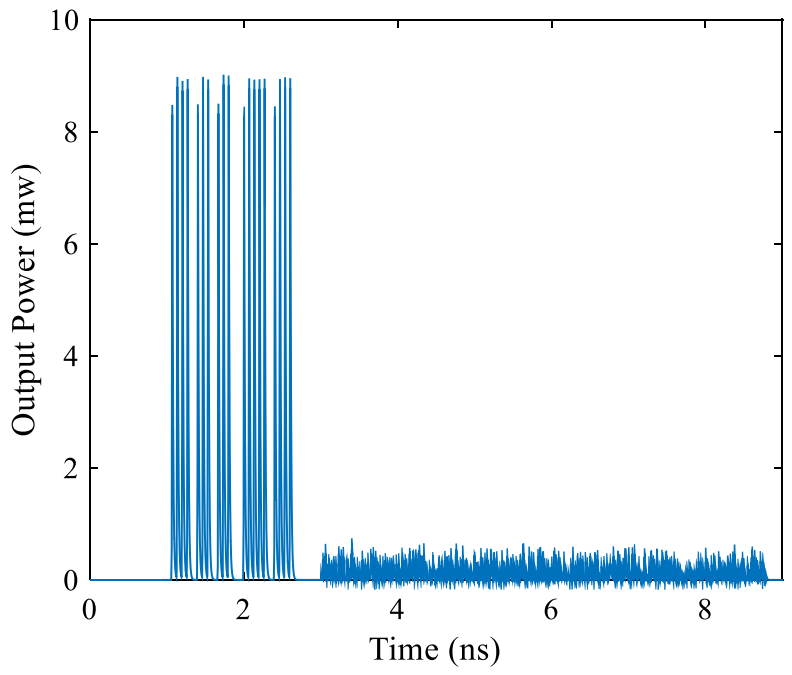

Figure 3. Output power packet extractor with 3-byte header and 128-byte payload input packet. 
Figure 4 demonstrates the output power of the port $\mathrm{C}$ with respect to the input data packet frequency. It can be seen that by increasing the bit rate, the output power decreases rapidly.

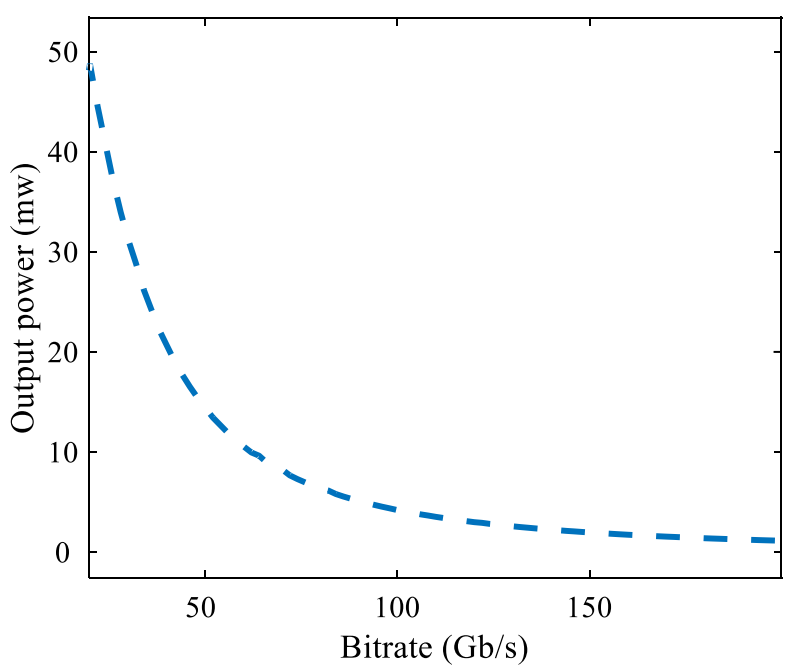

Figure 4. Output power of the port $\mathrm{C}$ with the varies Bit rate of input packet.

As mentioned before, if a packet with header bit rate of less than $20 \mathrm{~Gb} / \mathrm{s}$ and payload of more than $70 \mathrm{~Gb} / \mathrm{s}$ is applied to the proposed structure, a high-contrast header signal will appear at the output port.

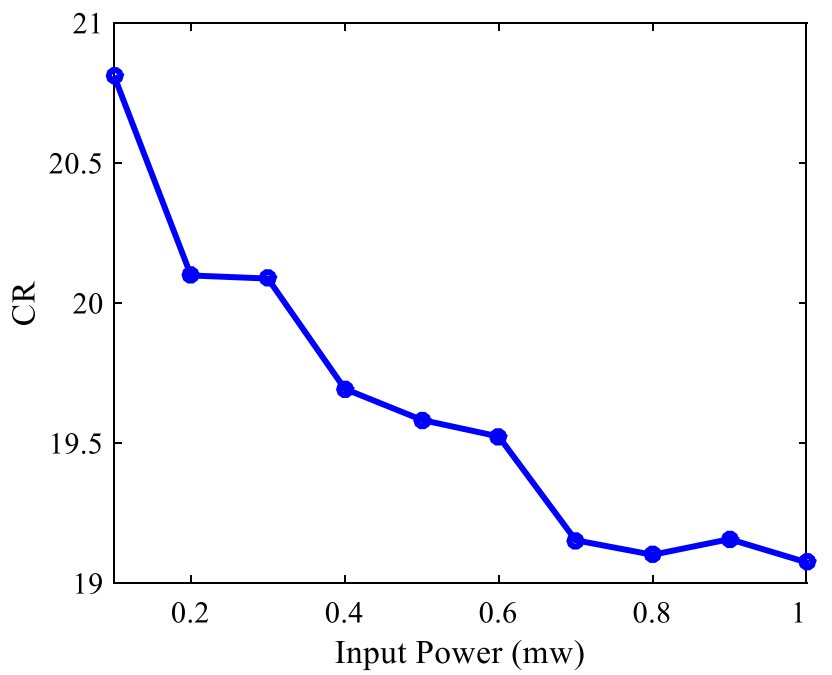

Figure 5. CR of header extractor vs changes of input packet signal power. 
Figure 5 demonstrates the varying of CR vs varying of input power. As can be seen by increasing the input power the CR decrease fast.

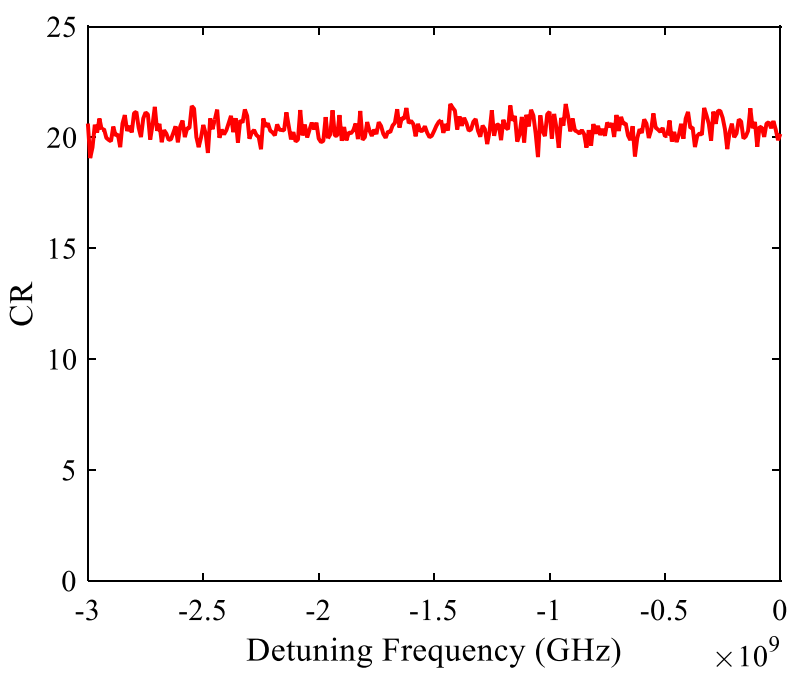

Figure 6. CR of header extractor vs packet input frequency detuning.

Figure 6 shows the CR vs header extractor vs packet input frequency detuning. As can be seen by varying of the input frequency detuning the CR doesn't change meaningful hence using of proposed structure it is possible to extract of headers with deferent frequencies.

\section{Conclusion}

In this paper, a novel structure based on ring laser was proposed to separate the header data stream. The proposed structure does not require synchronization and does not depend on the length of header. Simulation results show that output header CR can be larger than $20 \mathrm{~dB}$ when the input signal power is low as $0.2 \mathrm{~mW}$. On other way, CR increases as the current increases, while the required input power decreases. Also, by varying of the input frequency detuning the CR doesn't change meaningful. The structure is simple and optically integrable and can be used for the OPS networks. 


\section{Declarations}

Funding: The authors received no specific funding for this work.

Conflicts of interest/Competing interests:The authors declare that they have no conflict of interest.Availability of Data and Material: All the results are clearly mentioned in the article.

Author contributions: Dariush jafari; Proposed and simulated the structure. Also, analyzed data and co-wrote the paper. Mohammad Danaie , Pejman Rezaeia and Tofiq Nurmohammadi;

Supervised the research and co-wrote the paper.

Ethics approval: This material is the authors' own original work, which has not been previously published elsewhere. The paper is not currently being considered for publication elsewhere. The paper reflects the authors' own research and analysis in a truthful and complete manner. This research does NOT involve human participants or Animal.

Consent to Participate: All authors have been personally and actively involved in substantial work leading to the paper, and will take public responsibility for its content.

Consent to Publish: All authors have been personally and actively involved in substantial work leading to the paper, and consent to publish of submitted paper.

\section{References}

[1] Zuo C, Chen Q, Gu G, Feng S, Feng F, Li R, Shen G. High-speed three-dimensional shape measurement for dynamic scenes using bi-frequency tripolar pulse-width-modulation fringe projection. Optics and Lasers in Engineering. 2013 Aug 1;51(8):953-60.

[2] Zhang J, Sun J, Chen Q, Zuo C. Resolution analysis in a lens-free on-chip digital holographic microscope. IEEE Transactions on Computational Imaging. 2020 Jan 6;6:697-710.

[3] Zuo C, Sun J, Li J, Zhang J, Asundi A, Chen Q. High-resolution transport-of-intensity quantitative phase microscopy with annular illumination. Scientific reports. 2017 Aug 9;7(1):1-22.

[4] Hu Y, Chen Q, Feng S, Zuo C. Microscopic fringe projection profilometry: A review. Optics and Lasers in Engineering. 2020 Jul 1:106192.

[5] Zuo C, Chen Q, Tian L, Waller L, Asundi A. Transport of intensity phase retrieval and computational imaging for partially coherent fields: The phase space perspective. Optics and Lasers in Engineering. 2015 Aug 1;71:20-32.

[6] Li X, Feng Y, Liu B, Yi D, Yang X, Zhang W, Chen G, Liu Y, Bai P. Influence of NbC particles on microstructure and mechanical properties of AlCoCrFeNi high-entropy alloy coatings prepared by laser cladding. Journal of Alloys and Compounds. 2019 Jun 5;788:485-94.

[7] Geravand A, Danaie M, Mohammadi S. All-optical photonic crystal memory cells based on cavities with a dualargument hysteresis feature. Optics Communications. 2019 Jan 1;430:323-35.

[8] Danaee E, Geravand A, Danaie M. Wide-band low cross-talk photonic crystal waveguide intersections using selfcollimation phenomenon. Optics Communications. 2019 Jan 15;431:216-28. 
[9] Nurmohammadi T, Abbasian K, As'Adi MJ, Jafari D. Design of an ultrafast all-optical NOR logic gate based on Mach-Zehnder interferometer using quantum-dot SOA. Optik. 2014 Aug 1;125(15):4023-9.

[10] Hailu, Dawit Hadush. "Ring Optical Packet switched (OPS) network: Quality of Service (QoS) and traffic model." Optical Switching and Networking 28 (2018): 36-42.

[11] Meyer H, Sancho JC, Mrdakovic M, Miao W, Calabretta N. Optical packet switching in HPC. An analysis of applications performance. Future Generation Computer Systems. 2018 May 1;82:606-16.

[12] Lallas EN. A survey on key roles of optical switching and labeling technologies on big data traffic of Data Centers and HPC environments [J]. AIMS Electronics and Electrical Engineering. 2019 Jun 10;3(3):233-56.

[13] Qin J, Zhao Q, Yin H, Jin Y, Liu C. Numerical simulation and experiment on optical packet header recognition utilizing reservoir computing based on optoelectronic feedback. IEEE Photonics Journal. 2017 Jan 25;9(1):1-1.

[14] Miao W, de Waardt H, Calabretta N. Optical label switching add-drop multiplexer for high-performance metro networks. IEEE Photonics Technology Letters. 2016 Feb 11;28(10):1065-8.

[15] Wu Z, Li J, Tian Y, Ge D, Zhu P, Chen Y, Chen Z, He Y. Flexible-rate optical packet generation/detection and label swapping for optical label switching networks. Optical Fiber Technology. 2017 Mar 1;34:80-5.

[16] Calabretta N, Urban PJ, Geuzebroek DH, Klein EJ, de Waardt H, Dorren HJ. All-optical label extractor/eraser for in-band labels and 160-Gb/s payload based on microring resonators. IEEE Photonics Technology Letters. 2009 Feb 13;21(9):560-2.

[17] Golmohammadi S, Akbar Nejad V, Abbasian K, Rostami A. All-optical multi-wavelength header recognition using superimposed Bragg gratings based correlators. International Journal of Numerical Modelling: Electronic Networks, Devices and Fields. 2013 Jan;26(1):56-63.

[18] Liu HL, Bai XT, Chen CY. A novel scheme of all optical header extraction for optical packet switching network. Photonic Network Communications. 2011 Feb 1;21(1):34-8.

[19] Lallas EN. A survey on all optical label swapping techniques: Comparison and trends. Optical Switching and Networking. 2019 Jan 1;31:22-38.

[20] Hayashi D, Nakao K, Katayama T, Kawaguchi H. All-optical 2-bit header recognition and packet switching using polarization bistable VCSELs. Optics express. 2015 Apr 6;23(7):8357-64.

[21] Changliu N, Min Z, Peida Y. Header extraction with SOA-MZI in optical packet networks. In2009 2nd IEEE International Conference on Broadband Network \& Multimedia Technology 2009 Oct 18 (pp. 405-409). IEEE.

[22] Lu Y, Wang Y, Hu L, Du X, Li J, Li H, Bi M. Optical label switching based on Manchester code+ NRZ modulation. Optical Fiber Technology. 2021 Jan 1;61:102387.

[23] Kakarla R, Venkitesh D. Demonstration of optical header recognition for BPSK data using novel design of logic gates. Optics Communications. 2016 Mar 15;363:117-22.

[24] QUANG N, SÓNG ĐB. Multi-wavelength All-Optical Packet Switching Node Using Modified Pulse Position Modulation Header Processing.

[25]Zimmermann S, Wixforth A, Kotthaus JP, Wegscheider W, Bichler M. A semiconductor-based photonic memory cell. Science. 1999 Feb 26;283(5406):1292-5.

[26] Hill MT, Dorren HJ, De Vries T, Leijtens XJ, Den Besten JH, Smalbrugge B, Oei YS, Binsma H, Khoe GD, Smit MK. A fast low-power optical memory based on coupled micro-ring lasers. nature. 2004 Nov;432(7014):206-9. 
[27] Sorel M, Giuliani G, Scire A, Miglierina R, Donati S, Laybourn PJ. Operating regimes of GaAs-AlGaAs semiconductor ring lasers: experiment and model. IEEE Journal of Quantum Electronics. 2003 Sep 29;39(10):1187-95.

[28] Yuan G, Wang Z, Yu S. Dynamic switching response of semiconductor ring lasers to NRZ and RZ injection signals. IEEE Photonics Technology Letters. 2008 Apr 15;20(10):785-7.

[29] Born C, Sorel M, Yu S. Linear and nonlinear mode interactions in a semiconductor ring laser. IEEE Journal of quantum electronics. 2005 Feb 28;41(3):261-71.

[30] Sorel M, Laybourn PJ, Giuliani G, Donati S. Unidirectional bistability in semiconductor waveguide ring lasers. Applied Physics Letters. 2002 Apr 29;80(17):3051-3.

[31] Yuan G, Yu S. Bistability and switching properties of semiconductor ring lasers with external optical injection. IEEE Journal of Quantum Electronics. 2008 Jan;44(1):41-8.

[32] Yuan G, Wang Z, Li B, Memon MI, Yu S. Theoretical and experimental studies on bistability in semiconductor ring lasers with two optical injections. IEEE Journal of Selected Topics in Quantum Electronics. 2008 May;14(3):903-10.

[33] Chrostowski L, Shi W. Monolithic injection-locked high-speed semiconductor ring lasers. Journal of Lightwave Technology. 2008 Oct 1;26(19):3355-62. 
Figures

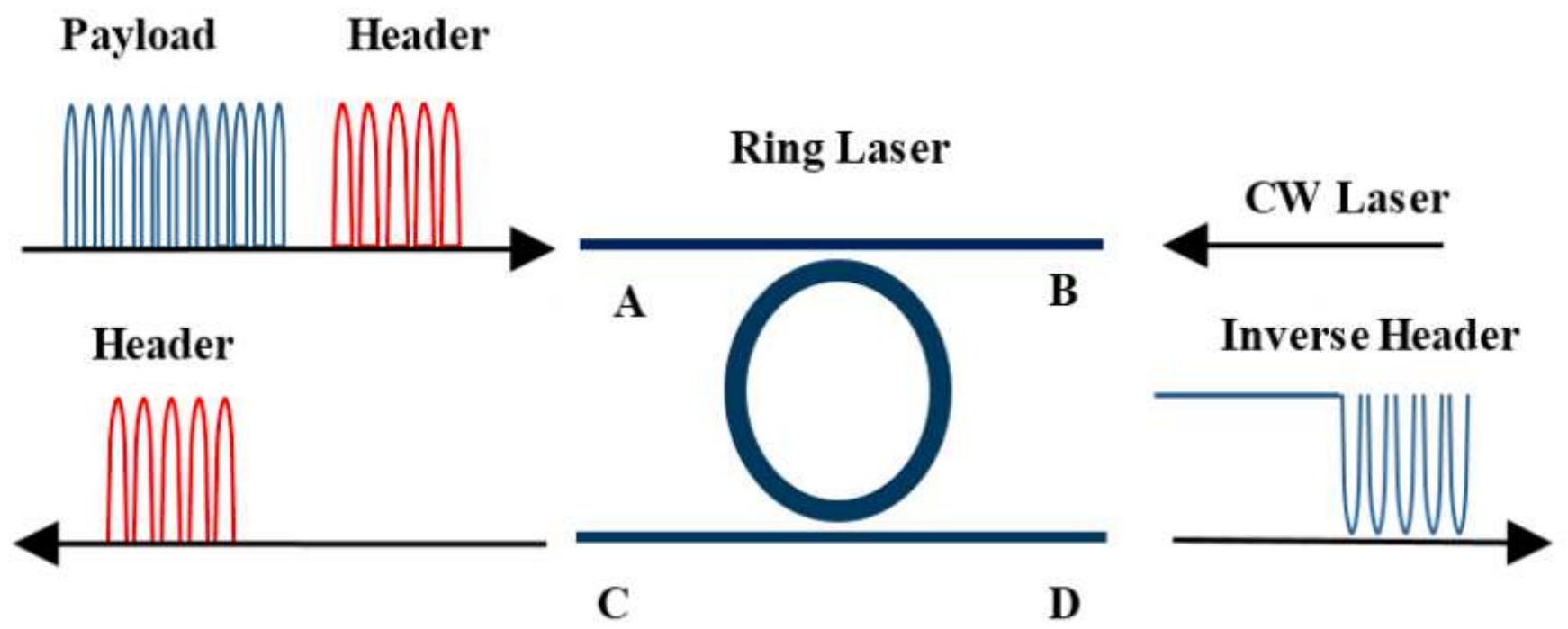

Figure 1

Schematic diagram of optical header extraction 


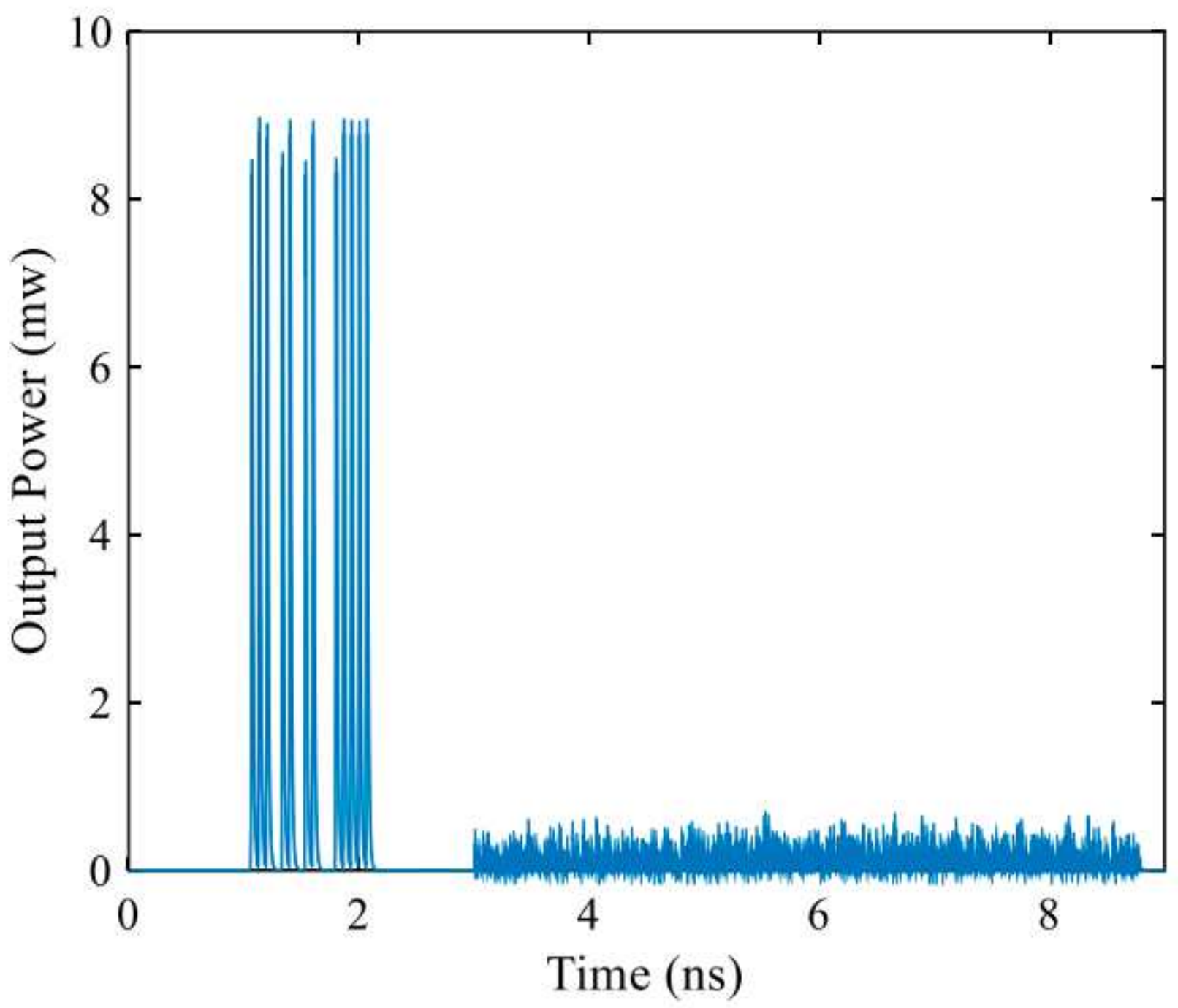

Figure 2

Output power packet extractor with 2-byte header and 128-byte payload input packet. 


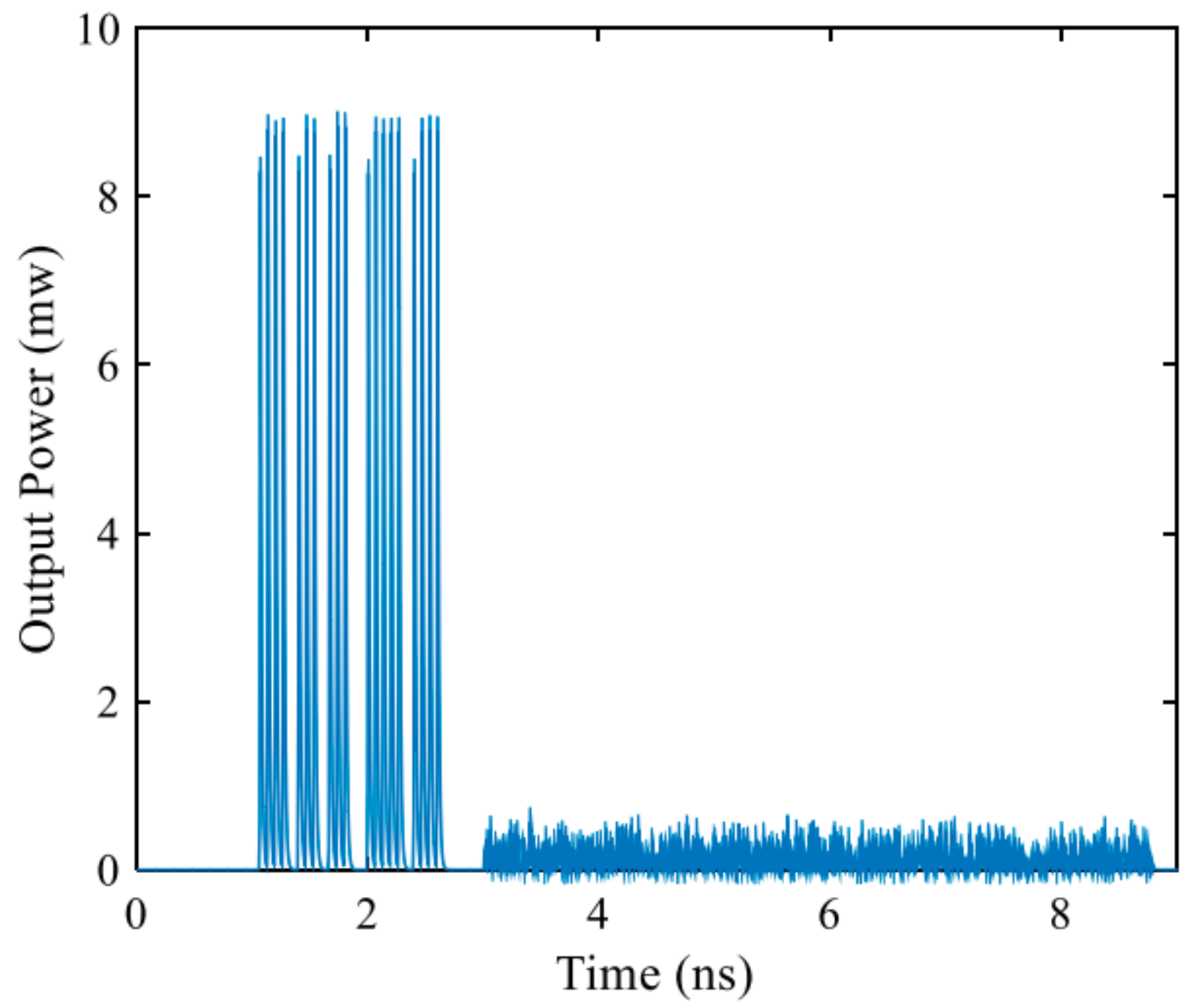

Figure 3

Output power packet extractor with 3-byte header and 128-byte payload input packet. 


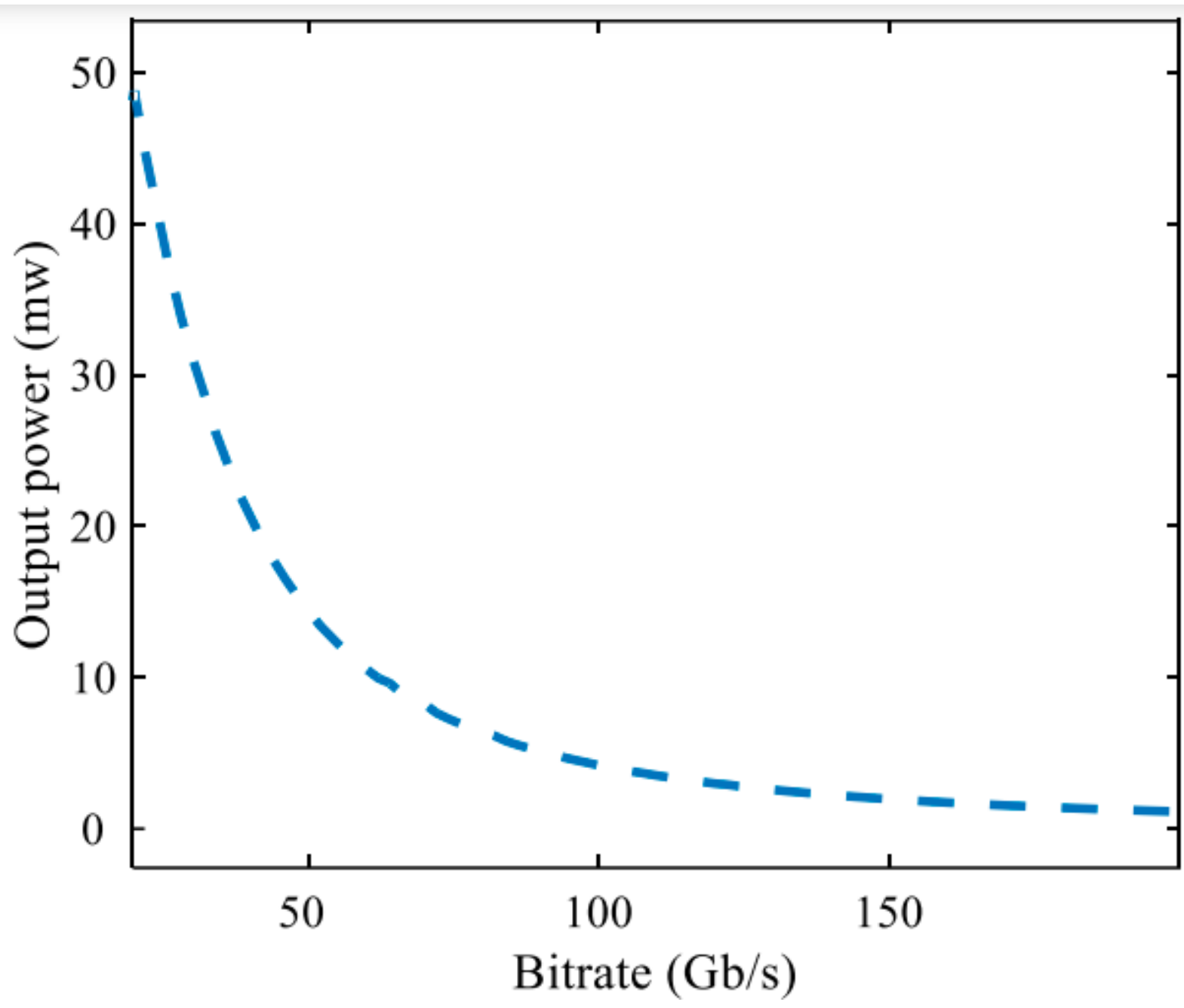

Figure 4

Output power of the port $\mathrm{C}$ with the varies Bit rate of input packet. 


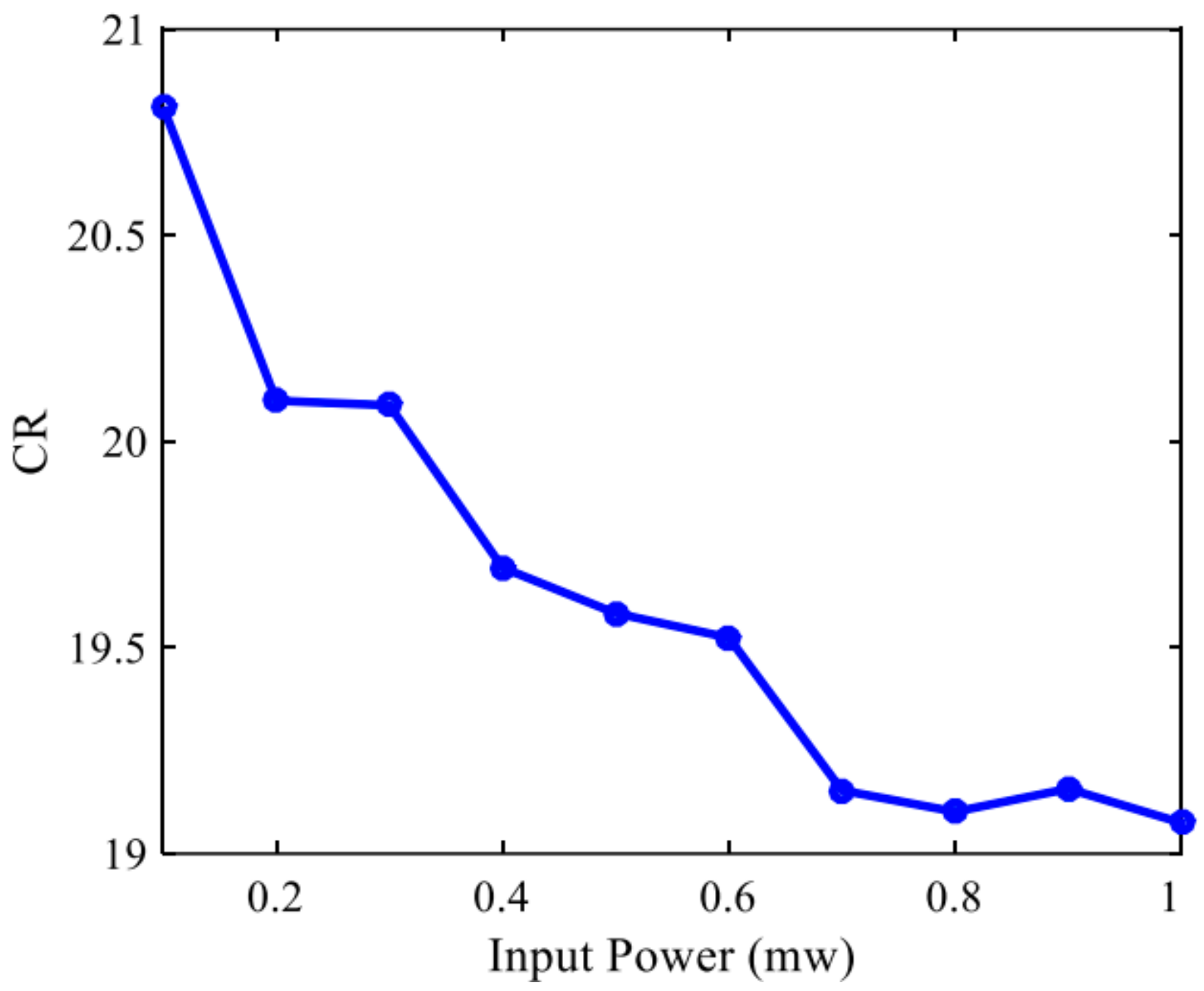

Figure 5

CR of header extractor vs changes of input packet signal power. 


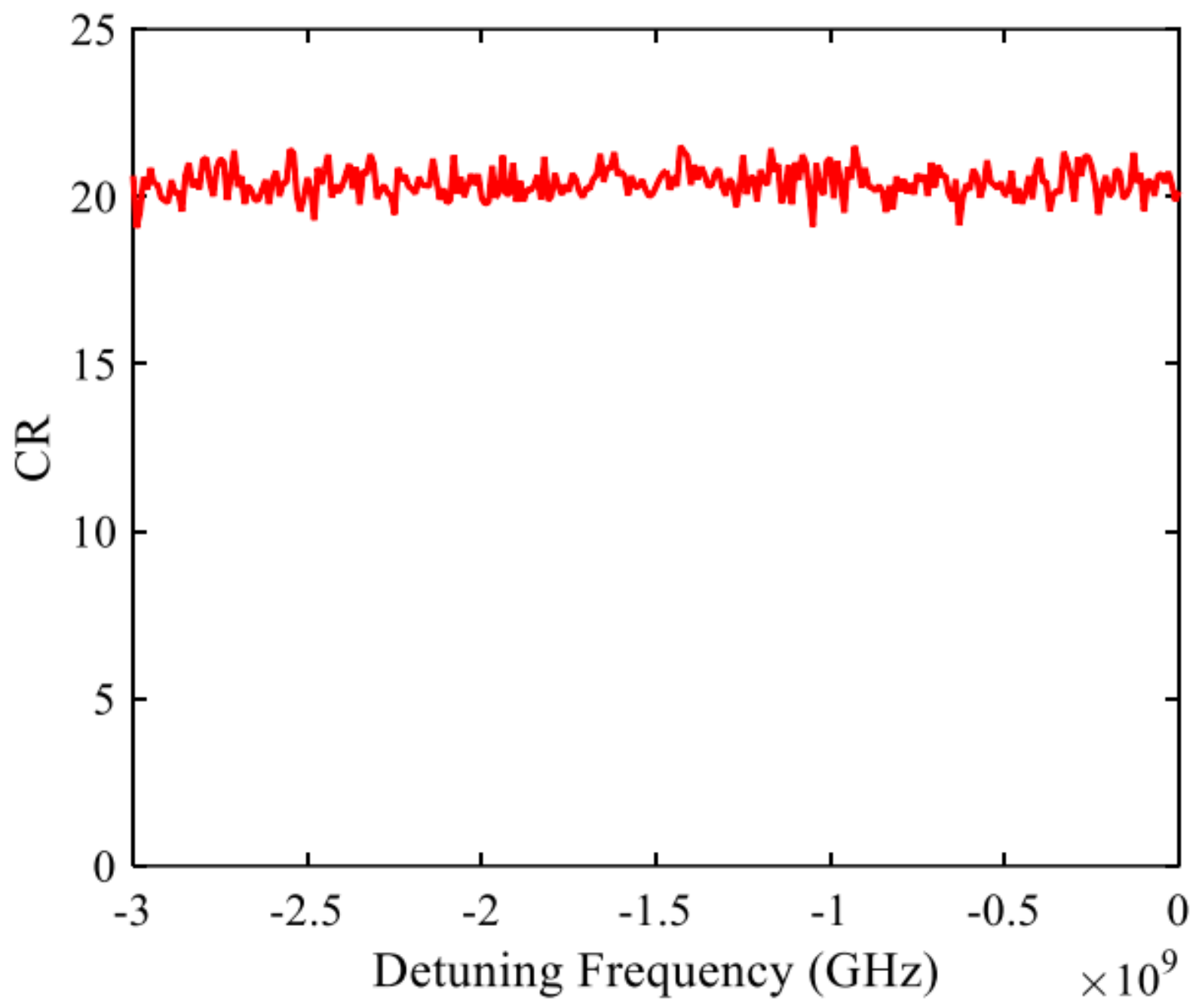

Figure 6

CR of header extractor vs packet input frequency detuning. 